\title{
Anwendung von Interferenz- und IR-Mikroskopie zur Charakterisierung und Untersuchung des Stofftransportes in nanoporösen Materialien
}

\author{
Lars Heinke \\ University of Leipzig \\ Follow this and additional works at: https://engagedscholarship.csuohio.edu/encbe_facpub

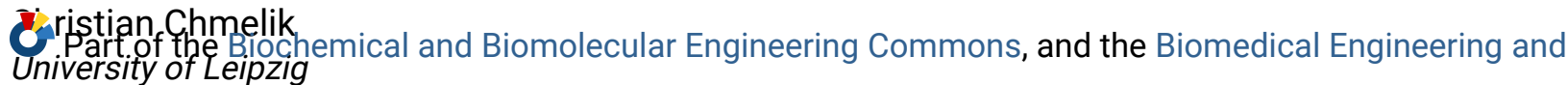 \\ Bioengineering Commons \\ Raveldeerstuneess to this work benefit you? Let us know!

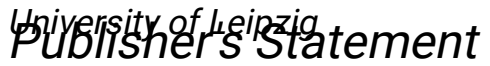 \\ \$erges the following article: Heinke, L.; Chmelik, C.; Kortunov, P.; \\ VasiensiowoSF/Rridlaven, D. \&.; Shah, D. \&.; Kärger, J. Anwendung von Interferenz- und IR- \\ Mikroskgpie zur Charakterisierung und Untersuchung des Stofftransportes in nanoporösen \\ Materialies: A A \\ form at [Link to final article]
}

See next page for additional authors

Original Citation

Heinke, L.; Chmelik, C.; Kortunov, P.; Vasenkov, S.; Ruthven, D. \&.; Shah, D. \&.; Kärger, J. Anwendung von Interferenz- und IR-Mikroskopie zur Charakterisierung und Untersuchung des Stofftransportes in nanoporösen Materialien. Chemie Ingenieur Technik 2007, 79, 1195-1203.

Repository Citation

Heinke, Lars; Chmelik, Christian; Kortunov, Pavel; Vasenkov, Sergey; Ruthven, Douglas M.; Shah, Dhananjai B.; and Kärger, Jörg, "Anwendung von Interferenz- und IR-Mikroskopie zur Charakterisierung und Untersuchung des Stofftransportes in nanoporösen Materialien" (2007). Chemical \& Biomedical Engineering Faculty Publications. 35. https://engagedscholarship.csuohio.edu/encbe_facpub/35

This Article is brought to you for free and open access by the Chemical \& Biomedical Engineering Department at EngagedScholarship@CSU. It has been accepted for inclusion in Chemical \& Biomedical Engineering Faculty Publications by an authorized administrator of EngagedScholarship@CSU. For more information, please contact library.es@csuohio.edu. 


\section{Authors}

Lars Heinke, Christian Chmelik, Pavel Kortunov, Sergey Vasenkov, Douglas M. Ruthven, Dhananjai B. Shah, and Jörg Kärger

This article is available at EngagedScholarship@CSU: https://engagedscholarship.csuohio.edu/encbe_facpub/35 


\title{
Anwendung von Interferenz- und IR-Mikroskopie zur Charakterisierung und Untersuchung des Stofftransportes in nanoporösen Materialien
}

\author{
Lars Heinke, Christian Chmelik, Pavel Kortunov, Sergey Vasenkov, \\ Douglas M. Ruthven, Dhananjai B. Shah und Jörg Kärger*
}

Herrn Professor Dr.-Ing. Jens Weitkamp zum 65. Geburtstag

\section{Einleitung}

Für viele industrielle Anwendungen von Zeolithen spielt der intrakristalline Stofftransport eine entscheidende Rolle $[1-6]$. Trotz des Wissens über die nicht-ideale Realstruktur der Zeolithe wird für die Beschreibung der Adsorptions- und Transporteigenschaften von Zeolithen aufgrund der beschränkten experimentellen Messmöglichkeiten gewöhnlich eine ideale innere Struktur angenommen. Dies reduziert die Aussagekraft der auf diesem Wege erzielten Ergebnisse, da diese Größen oft entscheidend von Strukturdefekten in den Kristallen geprägt sind. Für die Entwicklung neuer Materialien sowie für das genaue Verständnis der intrakristallinen Transportprozesse ist es entscheidend, den Einfluss der realen Zeolithstruktur zu kennen. Dieses Wissen ist zugleich auch zur Klärung der in vielen Fällen noch unbeantworteten Frage, warum die von verschiedenen experimentellen Methoden erhaltenen Ergebnisse für die intrakristalline Diffusion in gleichen Zeolithen um z. T. mehrere Größenordnungen voneinander abweichen, von ganz entscheidender Bedeutung [3,7-9].

Mit der Anwendung der Interferenz-Mikroskopie auf zeolithische Wirt-Gast-Systeme konnte erstmalig die zeitliche Entwicklung der intrakristallinen Konzentrationsprofile bei Änderung der umgebenden Gasphase verfolgt werden, insbesondere bei Adsorption und Desorption. Untersuchungen dieser Art sind an Zeolithen vom Typ LTA [10, 11], MFI [12 - 14] und AFI [14 - 19] durchgeführt worden. Viele dieser Kristalle sind speziell für unsere Untersuchungen im Labor von Professor Weitkamp am Institut für Technische Chemie an der Universität Stuttgart synthetisiert worden.

Ein besonders bemerkenswertes Beispiel, um die Vorzüge der Interferenzmikroskopie und ihre Vielseitigkeit darzustellen, liefert das Methanol-in-Ferrierit-System, das im Mittelpunkt dieses Beitrages steht. An diesem GastWirtsystem zeigt sich auch der Vorteil der Kombination der Interferenz-Mikroskopie (IFM) und der IR-Mikroskopie (IRM).

\section{Methoden und Experimentelles}

\subsection{Grundlagen der Interferenz- Mikroskopie}

Abb. 1 illustriert die Anwendung der Interferenz-Mikroskopie zur Untersuchung der Diffusion in nanoporösen Materialien. Die Technik basiert auf der Analyse der Interferenzmuster zweier Strahlen, wobei der eine durch den nanoporösen Kristall (gewöhnlich ein Zeolith [2, 3, 5, 20, 21]) und der andere durch die umgebende Gasatmosphäre verläuft.
Für viele industrielle Anwendungen von Zeolithen spielt der intrakristalline Stofftransport eine entscheidende Rolle.
Mit der Anwendung der InterferenzMikroskopie auf zeolithische WirtGast-Systeme konnte erstmalig die zeitliche Entwicklung der intrakristallinen Konzentrationsprofile bei Änderung der umgebenden Gasphase verfolgt werden. 
Abbildung 1. Darstellung der Interferenzmikroskopie. a) Zwei Lichtstahlen - einer passiert den Kristall mit der zweidimensionalen Porenstruktur und der andere passiert die umgebende Atmosphäre; b) Interferenzmikroskop; c) Interferenzmuster, das durch die unterschiedliche optische Dichte der von den beiden Lichtstrahlen passierten Medien erzeugt wird; d) Konzentrationsprofile, die aus den Änderungen der Interferenzmuster zu verschiedenen Zeiten berechnet wurden.

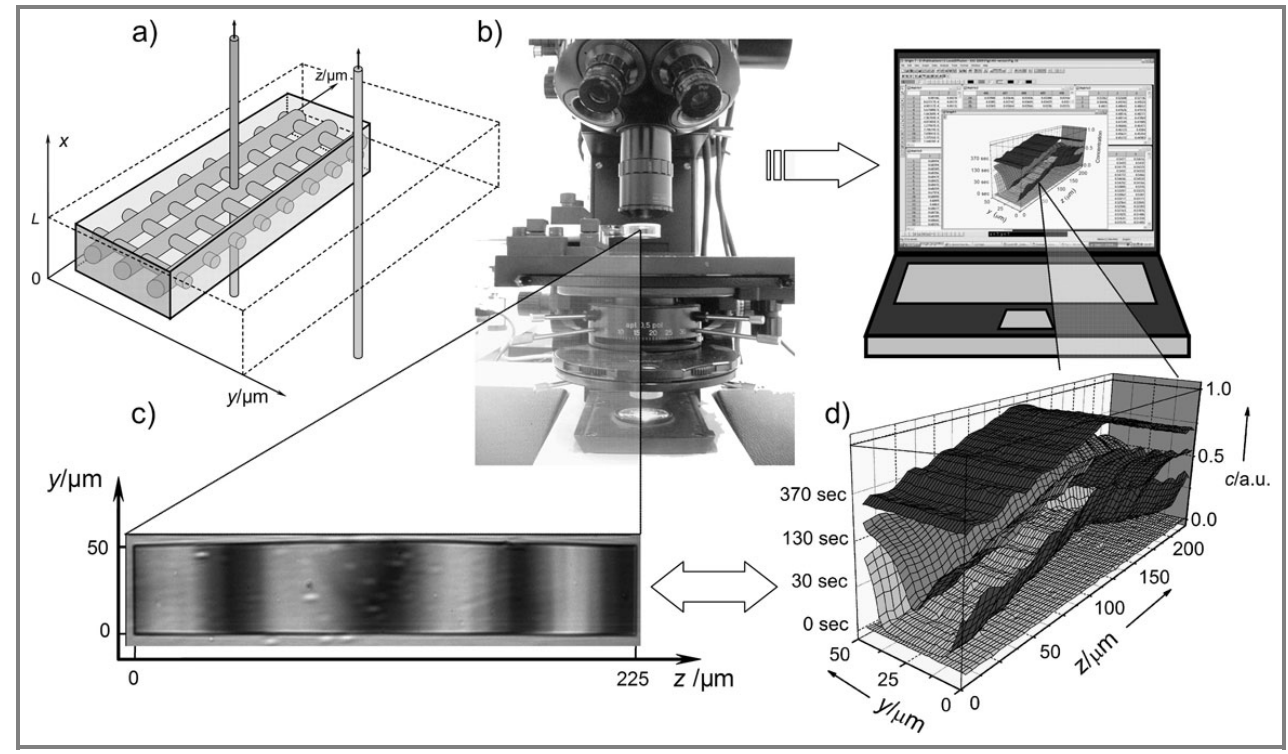

Da die optische Dichte von der Konzentration der Gastmoleküle abhängt, haben Änderungen in der lokalen Konzentration direkt Änderungen im Interferenzmuster zur Folge (s. Abbn. $1 \mathrm{~b}$ und c). Es ist daher möglich, die Konzentrationsprofile (s. Abb. 1d) aus den gemessenen Änderungen im Interferenzmuster abzuleiten.

Die direkt zugängliche Größe ist die intrakristalline Konzentration, in Strahlrichtung integriert ( $x$-Achse in Abb. 1a), mit einer Ortsauflösung von $\Delta y \times \Delta z \approx 0,5 \times 0,5 \mu \mathrm{m}^{2}$. Wenn auf Grund der Blockade der betreffenden Kristalloberflächen oder auf Grund der Architektur der Porenstruktur die Diffusion in $x$-Richtung ausgeschlossen ist, gibt es keine Veränderung der Konzentration in dieser Richtung. In diesem Fall liefert die Interferenzmikroskopie direkt die lokale Konzentration $c(y, z)$. Eine solche Situation liegt bei Materialien vor, deren Porensystem nur aus einer Anordnung paralleler Kanäle besteht (wie im Zeolithgerüst AFI [21]). Überraschenderweise sind jedoch diese Kanäle von inneren Barrieren versperrt [16, 22]. Die Interferenz-Mikroskopie hat gezeigt, dass solche inneren Transportwiderstände tatsächlich eine häufige Eigenschaft von vielen nanoporösen Zeolithen sind, darin eingeschlossen ist der wichtige Strukturtyp des MFI [15], mit ZSM-5 und Silikalit-1 als deren gebräuchlichste Vertreter $[13,21]$.

\subsection{Grundlagen der Infrarot- Mikroskopie (IRM)}

Die Integrationsprofile können auch auf eine etwas direktere Art gemessen werden, indem die Infrarot-Mikroskopie genutzt wird, um die IR-Absorptionsspektren der Gastmoleküle im
Kristall $\mathrm{zu}$ messen. Trotz der schlechten Ortsauflösung $(20 \mu \mathrm{m} \times 20 \mu \mathrm{m})$ ist die IR-Mikroskopie ein sehr nützliches Werkzeug, um die intrakristallinen Konzentrationsprofile zu untersuchen, da sie in der Lage ist, verschiedene Adsorbate zu unterscheiden. Dies wird dadurch ermöglicht, dass die Adsorbate $\mathrm{zu}$ charakteristischen IR-Absorptionsbanden zugeordnet werden können. Dadurch können Tracer-Austausch- sowie Counter- und CoDiffusionsexperimente durchgeführt werden [23-25].

\subsection{Untersuchtes Wirtssystem: Ferrierit}

Diese neue Methode der Diffusionsmessung wurde auf einen kationenfreien SiliciumFerrierit angewendet, der an der Universität Stuttgart synthetisiert worden ist [26]. Der untersuchte Zeolithkristall $(50 \mu \mathrm{m} \times 200 \mu \mathrm{m} \times$ $10 \mu \mathrm{m})$ hat die Form eines Quaders mit einem Dach auf den beiden großen Seitenflächen, wie es in Abb. 3a skizziert ist. Um auszuschließen, dass der Kristall noch organische Reststoffe enthält, wurde die Probe bei $700{ }^{\circ} \mathrm{C} 12 \mathrm{~h}$ einer Sauerstoffatmosphäre ausgesetzt und anschließend bei $400{ }^{\circ} \mathrm{C}$ unter Vakuum $12 \mathrm{~h}$ aktiviert, bevor das Adsorptions- und Desorptionsverhalten des Methanols untersucht wurde.

\subsection{Vakuumsystem und Durchführung}

Zur Messung wurden jeweils etwa 100 Kristalle in eine optische Vakuumküvette gefüllt und mit einem Vakuumsystem verbunden. Das Vakuumsystem besteht im Wesentlichen 
aus Vorratsvolumen, Drucksensoren und einer Turbomolekularpumpe. Das System erlaubt, den Gasdruck im System schnell, nahezu stufenförmig zu ändern oder den Druck konstant zu halten. Die Messungen der Konzentrationsintegrale werden immer an einem ausgewählten Kristall durchgeführt. Im Adsorptionsoder Desorptionexperiment wird der Druck in der Küvette zum Zeitpunkt Null erhöht oder erniedrigt, wogegen beim Tracer-AustauschExperiment die Gasphase zum Zeitpunkt Null ausgetauscht wird. Alle Messungen wurden bei Raumtemperatur durchgeführt.

\section{Ergebnisse}

\subsection{Form der Ferrierit-Kristalle und Besonderheiten bei der Sorbat- aufnahme}

Optische Untersuchungen hatten bereits erste Hinweise darauf geliefert, dass sich die untersuchten Ferrierit-Kristalle durch eine geometrische Besonderheit auszeichnen, nämlich dass die Dicke der Kristalle linear zur Mitte hin zunimmt. Mit dieser Annahme konnten die für das Sorptionsgleichgewicht gemessenen inhomogenen Konzentrationsprofile erklärt werden. Im Rahmen von Testmessungen für ein neues IR-Imaging-Mikroskop beim Hersteller (Bruker Optics) konnten durch Verwendung eines FPA-Detektors (Focal Plane Array) zwei-dimensionale Profile der Dicke einzelner Ferrierit-Kristalle gemessen werden. Dazu wurde die Fläche einer dem Zeolithen zuzuordnenden Bande des IR-Spektrums ausgewertet und als Graustufenkontrast abgebildet (s. Abb. 2a). Durch Vergleich mit den inhomogenen Konzentrationsprofilen im Sorptionsgleichgewicht konnte nun gezeigt werden, dass tatsächlich die Form der Kristalle die gemessenen Konzentrationsprofile erklären kann (s. Abb. 2b). Damit konnte geklärt werden, dass die beobachtete Ortsabhängigkeit der Sorbatkonzentration nicht, wie z. B. in [1419] für Kristalle der AFI-Struktur beschrieben, durch innere Barrieren bzw. Bereiche unterschiedlicher Sorptionskapazität, sondern einfach durch eine entsprechende Dickenänderung des Kristalls hervorgerufen wird.

Die Aufnahme der Konzentrationsprofile während der Ad- und Desorption brachte ein überraschendes Resultat: Entlang der größeren 10-Ring-Kanäle in $z$-Richtung konnten keine für Diffusion typischen Profile (gebogene Profile mit Abnahme zur Kristallmitte) gemessen werden, dafür wurden aber solche Profile entlang der schmaleren 8-Ring-Kanäle in $\gamma$-Richtung beobachtet (s. Abb. 3).

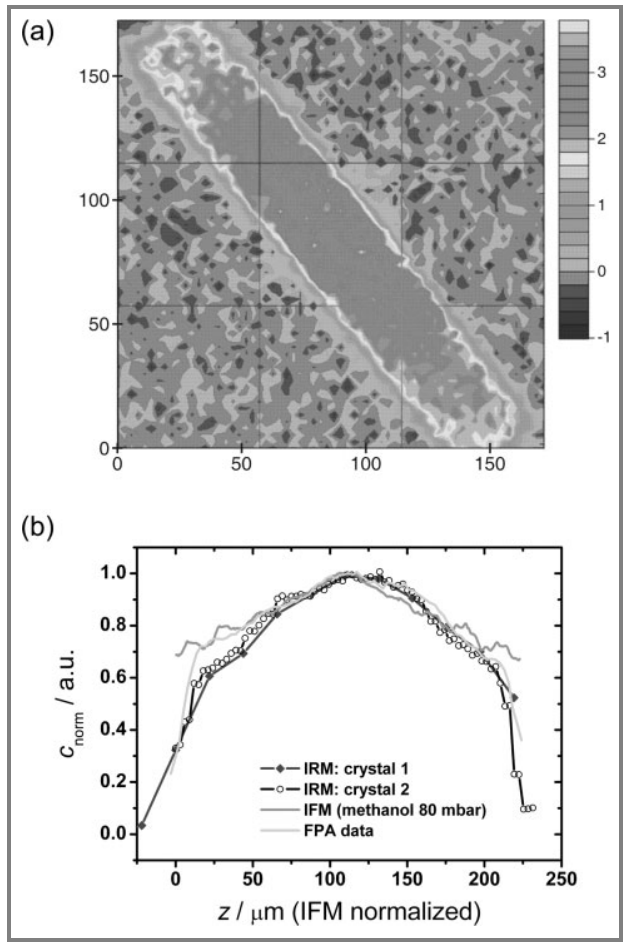

Abbildung 2. a) Dickeprofil eines Ferrierit-Kristalls, gemessen mit dem IR-Mikroskop HYPERION 3000 (Bruker Optics/Ettlingen). Im Graustufenkontrast dargestellt ist die Intensität einer Zeolithbande. b) Vergleich der eindimensionalen IRM-Dickeprofile mit einem IFM-Profil der Sorbatkonzentration im Sorptionsgleichgewicht (normiert). Dicke der Kristalle und Form des Konzentrationsprofils des Sorbates zeigen gute Übereinstimmung.

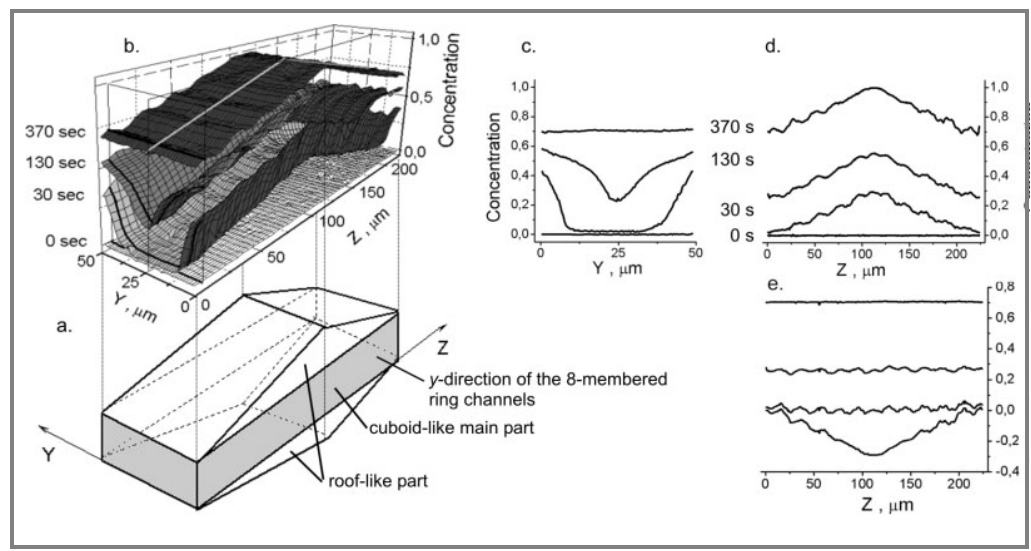

Abbildung 3. a) Skizze der Form der Ferrierit-Kristalle. b) 2D-Konzentrationsprofile für den gesamten Kristall. c) Konzentrationsprofile in $y$-Richtung nahe des Kristallrandes, $z=2 \mu \mathrm{m}$ ( $\equiv$ dicke schwarze Linie in den Profilen in b). d) Konzentrationsprofile in $z$-Richtung in der Kristallmitte, $y=25 \mu \mathrm{m}$ ( $\equiv$ helle Linie in den Profilen in b). e) Normalisierte Konzentrationsprofile in z-Richtung, erhalten durch Subtraktion der "Dachprofile". Die Konzentrationsprofile wurden mittels IFM für den Druckschritt $0 \rightarrow 80$ mbar aufgezeichnet, wobei die in a), b), c) und e) gezeigten Profile zu jeweils gleichen Zeiten nach Beginn der Adsorption gemessen wurden.

\subsection{Adsorptionsisotherme und Bestim- mung der absoluten Konzentration}

Zunächst wurde mittels IRM die Adsorptionsisotherme von Methanol in Ferrierit (Si-Form) gemessen. In Abb. 4 ist die gemessene Isotherme der Gleichgewichtskonzentration mit einem Datensatz aus der Literatur bei $303 \mathrm{~K}$ verglichen [27].

Eine direkte Bestimmung der absoluten Konzentration durch den Vergleich mit Literaturwerten [27] ist nicht sinnvoll, da die mittels 


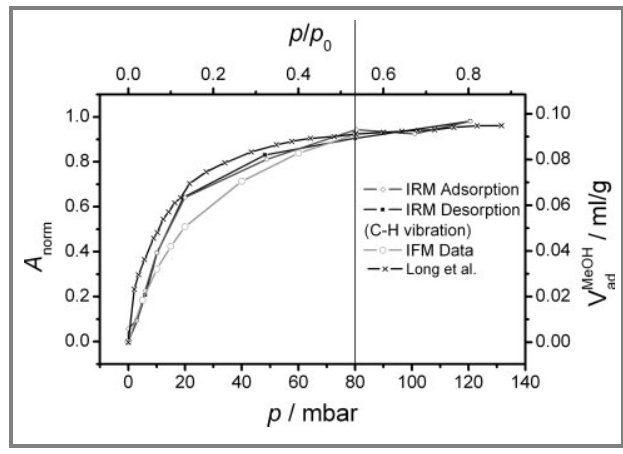

Abbildung 4. Isotherme von Methanol in Si-Ferrierit. Zum Vergleich wurde die Isotherme für Methanol in Si-Ferrierit aus [1] mit passender Skalierung ebenfalls dargestellt. Die Isotherme von Long et al. hat einen steileren Verlauf, was vermuten lässt, dass deren Kristalle nicht in reiner Si-Form vorlagen.

Stickstoffadsorption bestimmten Porenvolumina beider Kristallchargen deutlich voneinander abweichen (> $20 \%$ ). Jedoch konnte durch Vergleich der Bandenintensitäten des Signals des adsorbierten Methanols mit dem Signal der Gasphase unter Beachtung der geometrischen Abmessungen der IR-Zelle und den Strukturdaten des Ferrierits $[21,28,29]$ die intrakristalline absolute Konzentration direkt abgeschätzt werden. Der ermittelte Wert der maximalen Methanolbeladung von $V_{\mathrm{MeOH}}^{\max } \approx 0,175 \mathrm{~cm}^{3} / \mathrm{g}$ stimmt sehr gut mit dem über Stickstoffadsorption bestimmten Porenvolumen von $V_{\mathrm{N} 2}=$ $0,18 \mathrm{~cm}^{3} / \mathrm{g}$ überein. Diese Übereinstimmung bestätigt unsere Annahme, dass Methanolmoleküle auch in die 8-Ring-Kanäle eindringen.

Somit konnte nun bei IRM- und IFM-Messungen jedem Gasdruck eine Gleichgewichtskonzentration in absoluten Einheiten zugeordnet werden. Mit den Strukturdaten der Ferrierit-Kristalle und den Stoffparametern von Methanol kann die absolute Konzentration auch leicht in anderen Einheiten angegeben werden, wie z. B. in $\mathrm{mmol} \mathrm{g}^{-1}$ oder Molekülen pro Einheitszelle.

\subsection{Einfluss von intrakristalliner Diffusion und Oberflächenbarriere auf den Stofftransport}

Im Folgenden soll nun näher auf die transienten Konzentrationsprofile während verschiedener Druckschritte eingegangen werden [30]. Eine genauere Analyse der Sorptionsprofile zeigt, dass zwischen den Bereichen des Daches und dem quaderförmigen Hauptteil des Kristalls (s. Abb. 3a) unterschieden werden muss. Die gerade Form der Konzentrationsprofile entlang der $z$-Richtung (s. Abb. 3e) führt zunächst zu der Schlussfolgerung, dass - sofern überhaupt Stofftransport entlang der $z$-Richtung erfolgt - dieser durch Transportbarrieren an der äußeren Oberfläche limitiert ist. Tatsächlich konnte anhand der Konzentrationsprofile entlang der $\gamma$-Richtung nachgewiesen werden, dass für große Druckschritte $(0 \rightarrow$ 40 mbar und $0 \rightarrow 80$ mbar) ein kleiner Beitrag zur Gesamtbeladung entlang der $z$-Richtung erfolgt [31].

In $z$-Richtung wird die Form des Gleichgewichtsprofiles schon kurz nach Beginn der Sorption erreicht $(<30 \mathrm{~s}$ in Abb. 3e). Dies ist ein Zeichen dafür, dass die Sorbataufnahme in den Dachteil des Kristalls unmittelbar zu Beginn und damit also viel schneller als in den quaderförmigen Hauptteil des Kristalls erfolgt. Folglich müssen die Oberflächenbarrieren im Dachteil viel kleiner als im quaderförmigen Hauptteil sein. Dies lässt sich leicht mit den schrägen und somit vergrößerten Kanalöffnungen der 10-Ring-Kanäle im Dachteil erklären. Die große Geschwindigkeit der Sorbataufnahme im Bereich des Daches spricht dafür, dass in diesem Teil des Kristalls der Stofftransport entlang der größeren Kanäle dominiert.

Um das Sorptionsverhalten in dem quaderförmigen Teil des Kristalls einfacher analysieren zu können, wurden eindimensionale Profile entlang der $\gamma$-Richtung nahe dem Rand ausgewertet (s. Abb. 3c), wo die Dicke des Dachteiles und somit auch dessen Einfluss minimal ist. In diesen Experimenten wurde ein kleiner Druckschritt (5 - 10 mbar) genutzt, der im Henry-Bereich der Isotherme (s. Abb. 4) liegt, um eine Konzentrationsabhängigkeit der Diffusion auszuschließen. Die eindimensionalen Konzentrationsprofile entlang der $\gamma$-Richtung sind in Abb. 5 dargestellt. Wie bereits beschrieben, kann für kleine Drücke im quaderförmigen Teil des Kristalls die Sorbataufnahme entlang der $z$-Richtung als vernachlässigbar angesehen werden. Im Gegensatz zu dem Dachteil spricht hier die für intrakristalline Diffusion typische Krümmung der Profile entlang der $\gamma$-Richtung dafür, dass der Stofftransport entlang der 8-Ring-Kanäle erfolgt. Hierbei ist interessant, dass die Profile am Rand nicht sofort ihren Gleichgewichtszustand erreichen, wie bei alleinigem Einfluss intrakristalliner Diffusion zu erwarten wäre. Somit ist hier zusätzlich der Einfluss von Oberflächenbarrieren zu berücksichtigen. Es ist bekannt, dass bei makroskopischen Messverfahren (z. B. bei gravimetrischen Experimenten) thermische Effekte die Existenz einer Transportbarriere vortäuschen können. Es wurde jedoch gezeigt, dass für das vorliegende System thermische Effekte als Ursache der Oberflächenbarrieren ausgeschlossen werden können [14, 32]. 


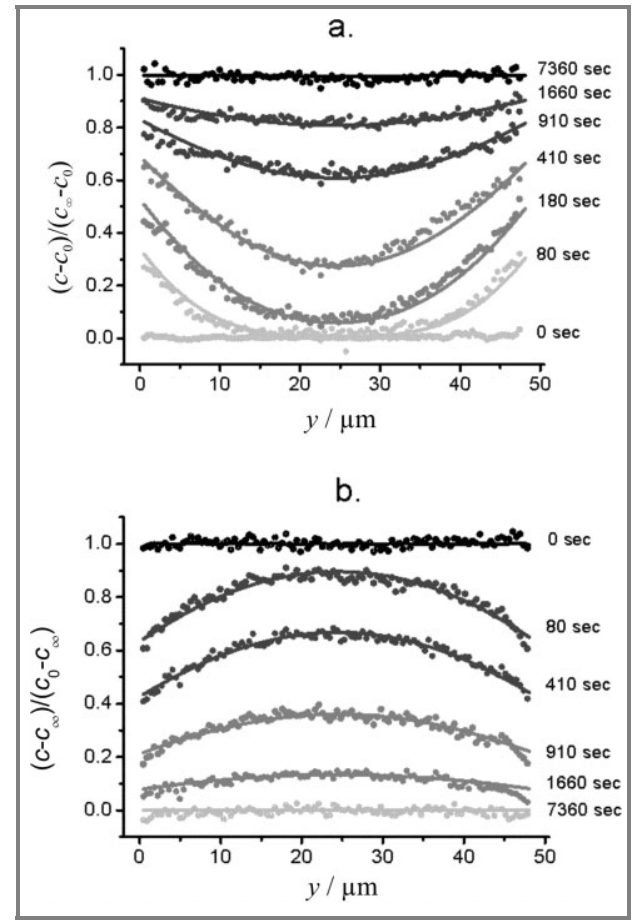

Abbildung 5. IFM-Konzentrationsprofile in $y$-Richtung für das System Methanol in Ferrierit bei a) Adsorption $5 \rightarrow 10$ mbar und b) Desorption $10 \rightarrow 5$ mbar. Die gemessenen Profile (Punkte) sind mit nach Gl. (1) berechneten Profilen (durchgezogene Linien) verglichen.

Entsprechend der Lösung der Diffusionsgleichung für diesen Fall [33]:

$\frac{c-c_{0}}{c_{\infty}-c_{0}}$
$=1-\sum_{\mathrm{n}=1}^{\infty} \frac{2 L \cos \left(\beta_{\mathrm{n}} x / l\right) \exp \left(-\beta_{\mathrm{n}}^{2} D t / \ell^{2}\right)}{\beta_{\mathrm{n}}^{2}\left(\beta_{\mathrm{n}}^{2}+L^{2}+L\right)}$

mit $L \equiv \frac{\ell a}{D}=\beta_{\mathrm{n}} \tan \beta_{\mathrm{n}}$

konnten die Profile der Ad- und Desorption mit Werten für $D=8,2 \cdot 10^{-13} \mathrm{~m}^{2} / \mathrm{s}$ und $a=4,2 \cdot 10^{-8} \mathrm{~m} / \mathrm{s}$ ( $D$ - Diffusionskoeffizient, $a-$ Permeabilität der Oberflächenbarrieren) in guter Übereinstimmung angepasst werden (s. Abb. 5).

Dass die Profile für die Ad- und Desorption mit den gleichen Werten von $D$ und $a$ durch Gl. (1) beschrieben werden können, ist trotz des Arbeitens im Henry-Bereich nicht unbedingt zu erwarten, da auch der Einfluss der Konzentrationsabhängigkeit der Permeabilität der Oberflächenbarrieren beachtet werden muss. So unterschied sich z. B. für die Druckstufe $0 \rightarrow 5$ mbar die Form der Profile der Adsorption und Desorption deutlich voneinander. Eine Anpassung nach Gl. (1) war formal nur für die Desorptionsprofile möglich, wobei aber keine für alle Zeiten passenden Parameter $D$ und $a$ gefunden werden konnten. Da dies aber notwendige Bedingung für die Gültigkeit von Gl. (1) ist, muss davon ausgegangen werden, dass die zugrunde liegende Theorie den Sorptionsvorgang hier nur unzureichend beschreibt. Auf die Konzentrationsabhängigkeit der Diffusion und Oberflächenpermeabilität wird in Abschnitt 3.4 näher eingegangen.

Unser Modellvorschlag für den gefundenen Transportwiderstand ist eine relativ dünne Schicht an der Oberfläche mit stark verlangsamtem Stofftransport (z. B. wegen sterischer Behinderungen, verursacht durch vermehrte Strukturdefekte in der oberflächennahen Schicht).

Neben der Zielstellung, den Einfluss der Realstruktur der Zeolithe auf den Stofftransport direkt zu untersuchen, sind die Ergebnisse auch unter der noch immer bestehenden Fragestellung interessant, warum die mit unterschiedlichen Methoden für das gleiche System gemessenen Diffusionskoeffizienten um z. T. mehrere Größenordnungen voneinander abweichen [3, 7 -9]. Dazu wurden die über den gesamten Kristall gemessenen Sorptionskurven ausgewertet (s. Abbn. 6 und 7). Diese Daten entsprechen den Ergebnissen, die mit einem makroskopischen Messverfahren gewonnen würden.

Bemerkenswert ist hier, dass bis auf einen kleinen Zeitverzug die Sorptionskurven einem

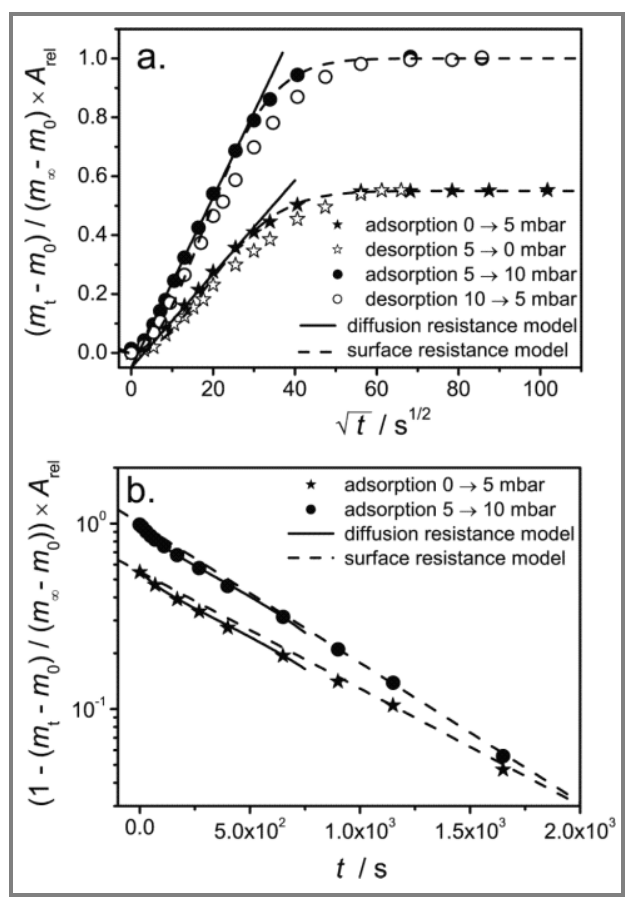

Abbildung 6. IFM Sorptionskurven, gemessen für den gesamten Kristall für verschiedene Druckschritte. Ebenfalls eingezeichnet sind die theoretischen Kurven, die durch Anpassung nach GI. (2) ("diffusion resistance model“) erhalten wurden. Um eine Darstellung im Diagramm zu ermöglichen, wurden die Sorptionskurven für 5 mbar jeweils auf die relative Beladung normiert. 


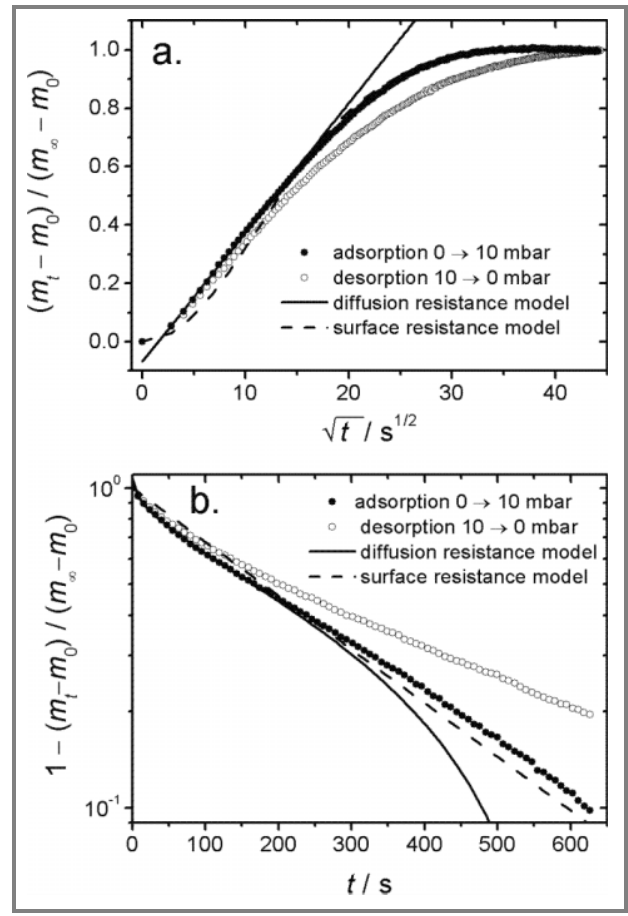

Abbildung 7. IRM Sorptionskurven, gemessen für den gesamten Kristall für verschiedene Druckschritte $(0 \rightarrow 10$ und $10 \rightarrow 0$ mbar). Ebenfalls eingezeichnet sind die theoretischen Kurven, die durch Anpassung nach GI. (2) ("diffusion resistance model") erhalten wurden.

für intrakristalline Diffusion typischen Verlauf folgen (Kurzzeit-Darstellung s. Abbn. 6 und 7a: linear bis etwa 0,7; Langzeit-Darstellung s. Abbn. 6 und 7b: asymptotisches Verhalten für große Zeiten mit rückverlängertem Achsenschnittpunkt bei ca. 0,8 für $t=0$ ).

Ohne Kenntnis der Konzentrationsprofile bestünde der nächstliegende Weg der Datenanalyse in einer Anpassung nach der Lösung der Diffusionsgleichung für Diffusion in einer planparallelen Platte [33]:

$$
\begin{aligned}
& \frac{m_{\mathrm{t}}-m_{0}}{m_{\infty}-m_{0}} \\
& =1-\frac{8}{\pi^{2}}{ }_{\mathrm{n}=0}^{\infty} \frac{\exp \left[-(2 n+1)^{2} \pi^{2} D t / 4 \ell^{2}\right]}{(2 n+1)^{2}}
\end{aligned}
$$

Entsprechend müsste angenommen werden, dass der Stofftransport im Wesentlichen entlang der größeren 10-Ring-Kanäle erfolgt. Durch Anpassung der IFM-Daten für den Druckschritt $5 \rightarrow 10$ mbar nach Gl. (2) erhält man für den Diffusionskoeffizient einen Wert von $D_{\mathrm{Pl}}^{\mathrm{z}}=1,5 \cdot 10^{-12} \mathrm{~m}^{2} / \mathrm{s}$. Dieser Wert weicht um fast zwei Größenordnungen von dem aus den Konzentrationsprofilen ermittelten Wert $\mathrm{ab}$ !

Somit konnte gezeigt werden, dass in diesem Fall ohne die Kenntnis der Konzentrationsprofile erhebliche Unsicherheiten bezüg- lich des für die Datenanalyse geeigneten Modells bestehen. Es sei an dieser Stelle explizit darauf hingewiesen, dass makroskopische Messverfahren, die zur Charakterisierung der Transporteigenschaften von Zeolithen vielfach angewendet werden, diese Information nicht liefern können. Die Ergebnisse dieser Messungen sind daher nur korrekt, wenn das angenommen Diffusionsmodell das tatsächliche Verhalten des Systems richtig beschreibt.

\subsection{Mikroskopische Bestimmung der Diffusionskoeffizienten}

Bisher wurden zur Berechnung der Transportparameter spezielle Lösungen der Diffusionsgleichungen verwendet. Jedoch sind mit jeder Lösung spezielle Randbedingungen verknüpft, die in der Realität nicht immer erfüllt sind, z. B. Form der Kristalle, konzentrationsunabhängiger Diffusionskoeffizient. Einige Probleme, die bei der Verwendung spezieller Lösungen auftreten können, wurden bereits im vorangegangenen Abschnitt dargestellt. Ideal wäre dagegen ein Verfahren, dass eine direkte Bestimmung der relevanten Transportgrößen aus den transienten Konzentrationsprofilen gestattet. Die Grundlage für eine entsprechende Analyse der Konzentrationsprofile bildet das 2. Fick'sche Gesetz für eindimensionale Diffusion entlang der $\gamma$-Richtung [31, 34]:

$\frac{\partial c(\gamma, t)}{\partial t}=\frac{\partial}{\partial \gamma}\left(D(c(\gamma, t)) \frac{\partial c(\gamma, t)}{\partial \gamma}\right)$

$=\frac{\partial D(c(\gamma, t))}{\partial c}\left(\frac{\partial c(\gamma, t)}{\partial \gamma}\right)^{2}+D(c(\gamma, t)) \frac{\partial^{2} c(\gamma, t)}{\partial \gamma^{2}}$

mit $D$ als Transportdiffusionskoeffizienten. Dabei hängt die Konzentration $c$ im Kristall nur von der Zeit $t$ und der Raumkoordinate $\gamma$ $\mathrm{ab}$. Da diese Informationen in den transienten IFM-Profilen enthalten sind, ist die Bestimmung des Diffusionskoeffizienten auf diesem Wege prinzipiell möglich.

Da der erste Term auf der rechten Seite in Gl. (3) aus Symmetriegründen in der Mitte der Kristalle verschwindet, wurden zunächst die Konzentrationsprofile dort analysiert, um eine erste Abschätzung der Konzentrationsabhängigkeit des Diffusionskoeffizienten zu erhalten. Danach wurde dieser Zusammenhang durch Anpassung der kompletten Profile aller Druckschritte verifiziert und die Genauigkeit weiter verbessert. Wie in [31] beschrieben ist, wird die Permeabilität der Oberfläche bestimmt, indem der Fluss durch die Kristalloberfläche mit dem Konzentrationsunterschied zwischen dem momentanen Wert und dem Gleichgewichtswert an der Oberfläche in Beziehung gesetzt wird. 
Schließlich wurden die gemessenen Konzentrationsprofile mit den simulierten Profilen verglichen, die aus der numerischen Lösung des 2. Fick'schen Gesetzes mit den ermittelten Diffusionskoeffizienten und Oberflächenpermeabilitäten erhalten wurden. Dabei wurde der Algorithmus der „Finite Difference Solution" angewendet. Die Übereinstimmung mit den gemessenen Profilen war für alle Druckschritte exzellent.

Als Beispiel ist der Vergleich zwischen gemessenen und simulierten Profilen für den Druckschritt 0 - 5 mbar in Abb. 8 dargestellt. In Abb. 9 ist die in der Mitte der Profile ermittelte Konzentrationsabhängigkeit des Diffusionskoeffizienten (für alle gemessenen Druckschritte) mit der Abhängigkeit verglichen, die für den Vergleich mit den gemessenen Profilen in der „Finite Difference Solution“ (s. Abb. 8) verwendet wurde.

Um den Vorteil der hohen Orts- und Zeitauflösung der IFM-Profile zu nutzen, wurde der Diffusionskoeffizient direkt auf mikroskopischem Weg ohne Modellannahmen berechnet. Die Transport bestimmenden Parameter konnten direkt aus den gemessenen Profilen abgeleitet werden. Solche detaillierte Informationen können derzeit mit keinem anderen experimentellen Verfahren gewonnen werden. Dies unterstreicht einmal mehr das große Potenzial der angewandten Methode der Interferenz-Mikroskopie zur Charakterisierung des Stofftransportes in nanoporösen Medien.

\section{Zusammenfassung}

Die Leistungsfähigkeit der Methode der Interferenz-Mikroskopie zur Untersuchung des Stofftransportes an nanoporösen Materialien wurde am Beispiel des Sorptionsverhaltens von Methanol an Ferrierit-Einkristallen illustriert. Wertvolle ergänzende Information zur Kristallgeometrie und zur Sorbatverteilung wurden durch Paralleluntersuchungen mittels IR-Mikroskopie zugänglich. Die Methode der Interferenz-Mikroskopie ermöglicht es, transiente intrakristalline, am einzelnen Kristall gemessene Konzentrationsprofile mit hoher Ortsauflösung aufzunehmen. Diese detaillierten Informationen über den Transportprozess sind notwendig, um Richtung und Geschwindigkeit des Stofftransportes eindeutig bestimmen zu können und um die Existenz sowie den möglichen Einfluss struktureller Defekte oder Oberflächenbarrieren nachweisen und quantifizieren zu können.

Solch detaillierte Angaben zum Transportgeschehen können mittels makroskopischer Verfahren, wie sie zur Charakterisierung der

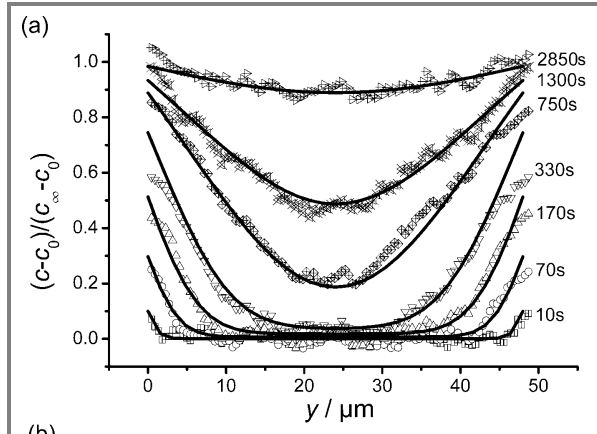

(b)

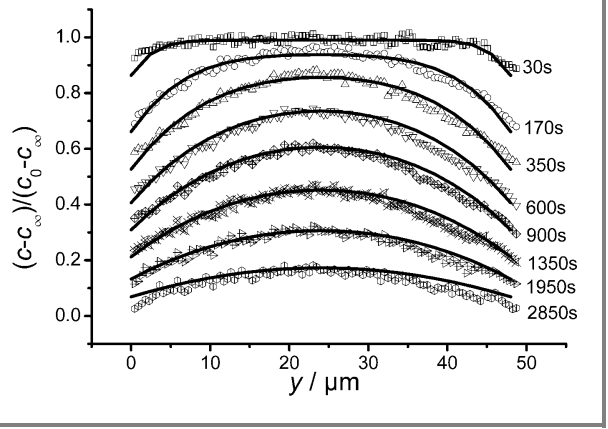

Abbildung 8. Vergleich von simulierten und experimentellen Profilen für den Druckschritt $0 \rightarrow 5$ mbar (a) und $5 \rightarrow 0$ mbar (b). Die Punkte repräsentieren die gemessenen IFM- Profile. Die durchgezogenen Linien wurden mit der „Finite Difference Solution" und einem Diffusionskoeffizienten simuliert, der in Abb. 9 abgebildet ist.

Transporteigenschaften von Zeolithen breite Anwendung finden, nicht erhalten werden. Für das untersuchte System wurde gezeigt, dass in diesem Fall die Interpretation der Messdaten leicht zu falschen Schlussfolgerungen bezüglich des Stofftransportes führen kann. Wenn die für ein System mittels mikroskopischer und makroskopischer Verfahren er-

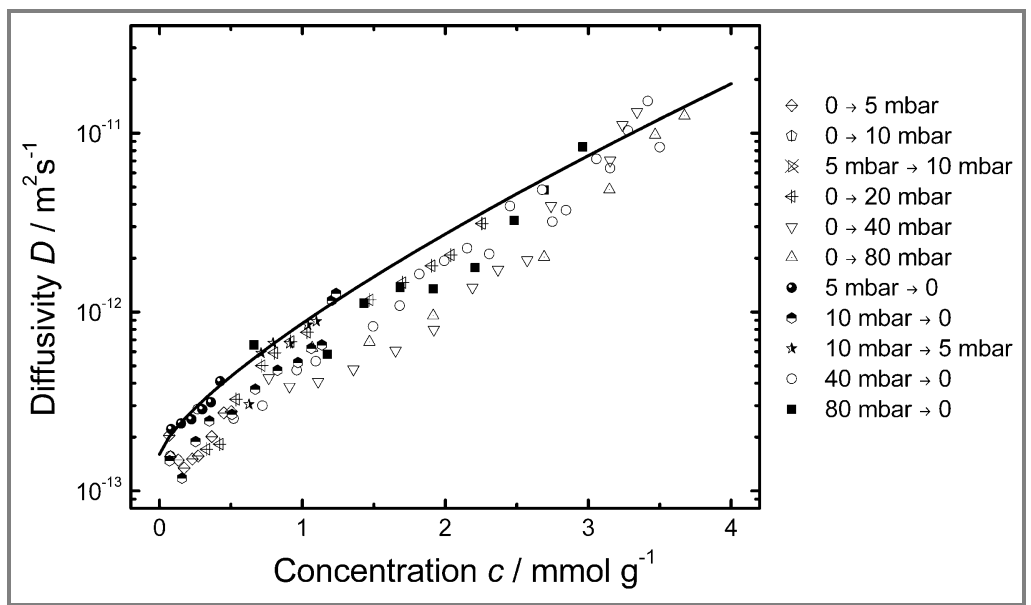

Abbildung 9. Konzentrationsabhängigkeit des Transportdiffusionskoeffizienten in $y$-Richtung, ermittelt aus mehreren Druckschritten für die Mitte des Kristalls. Die durchgezogene Linie stellt die Konzentrationsabhängigkeit dar, die für die Anpassung der transienten Profile der jeweiligen Druckschritte verwendet wurde (z. B. Druckschritt 0-5 mbar in Abb. 8). 


\section{Die Interferenz- Mikroskopie bietet einen Zugang zu einzigartigen Infor- mationen, die derzeit mit keiner anderen experimen- tellen Methode gewonnen werden können.}

mittelten Diffusionskoeffizienten verglichen werden sollen, ist daher ein genaues Wissen um strukturelle Besonderheiten und um den Einfluss von Transportbarrieren an der Oberfläche unerlässlich.

Mit der im letzten Abschnitt vorgestellten Methode der mikroskopischen Bestimmung lokaler Diffusionskoeffizienten kann der Stofftransport in den untersuchten Systemen einschließlich des Einflusses der Realstruktur umfassend beschrieben und verstanden werden, ohne dass dabei Modellannahmen zugrunde gelegt werden müssen. Die Interferenz-Mikroskopie bietet somit einen Zugang $\mathrm{zu}$ einzigartigen Informationen, die derzeit mit keiner anderen experimentellen Methode gewonnen werden können. Damit hat sie das Potenzial, einen fundamentalen Beitrag zum Verständnis von Transportprozessen in nanoporösen Materialen zu liefern.

Diese Arbeit wurde im Rahmen des internationalen Graduiertenkollegs „Diffusion in Porous Materials“ und der internationalen Forschergruppe „Diffusion in Zeolites“ mit Unterstützung der Deutschen Forschungsgemeinschaft sowie der Max-Buchner-Forschungsstiftung durchgeführt. Wir widmen sie Herrn Professor Dr.-Ing. Jens Weitkamp in Dankbarkeit für vielfältige Unterstützung, insbesondere für die Bereitstellung des Zeolithmaterials für unsere Arbeiten, sein förderndes Interesse sowie zahlreiche wertvolle Hinweise.

\section{Heinke, \\ C. Chmelik, \\ P. Kortunov, \\ J. Kärger}

(kaerger@physik.uni-leipzig.de),

Fakultät für Physik und Geowissenschaften,

Universität Leipzig, Linnéstraße 5,

D-04103 Leipzig, Germany;

P. Kortunov,

Corporate Strategic Research, ExxonMobil Research and Engineering Company, Annandale, New Jersey 08801, USA;

S. Vasenkov,

Chemical Engineering Department, University of Florida, PO Box 116005 Gainesville,

FL 32611-6005, USA;

\section{M. Ruthven,}

Department of Chemical \& Biological Engineering, University of Maine, 5737 Jenness Hall,

Orono, Maine 04469-5737, USA;

D. B. Shah,

Department of Chemical a Biomedical Engineering, Cleveland State University,

2121 Euclid Avenue, Cleveland, Ohio 44115, USA.

\section{Literatur}

[1] G. Ertl, H. Knötzinger, J. Weitkamp, Handbook of Heterogeneous Catalysis, Wiley-VCH, Chichester 1997.

[2] F. Schüth, K. S. W. Sing, J. Weitkamp, Handbook of Porous Solids, Wiley-VCH, Weinheim 2002.

[3] J. Kärger, D. M. Ruthven, Diffusion in Zeolites and Other Microporous Solids, Wiley \& Sons, New York 1992.

[4] J. Kärger, S. Vasenkov, S. M. Auerbach, Diffusion in Zeolites, in Handbook of Zeolite Science and Technology (Eds: S. M. Auerbach, K. A. Carrado, P. K. Dutta), Marcel Dekker, Inc., New York, Basel 2003, 341-423.

[5] N. Y. Chen, T. F. Degnan, C. M. Smith, Molecular Transport and Reaction in Zeolites, Wiley \& Sons, New York 1994.

[6] F. Keil, Diffusion und Chemische Reaktion in der Gas/Feststoff-Katalyse, Springer-Verlag, Berlin 1999.

[7] H. Jobic, J. Kärger, C. Krause, S. Brandani, A. Gunadi, A. Methivier, G. Ehlers, B. Farago, W. Haeussler, D. M. Ruthven, Diffusivities of n-Alkanes in 5A Zeolite measured by Neutron Spin Echo, Pulsed-Field Gradient NMR, and Zero Length Column techniques, Adsorption 2005, 11, 403-407.

[8] J. Kärger, Measurement of Diffusion in Zeolites - A Never Ending Challenge?, Adsorption 2003, 9, 29-35.

[9] J. Kärger, Diffusion under Confinement, Sitzungsberichte der Sächsischen Akademie der Wissenschaften zu Leipzig 2003, 128 (6), 5-43.

[10] U. Schemmert, J. Kärger, J. Weitkamp, Interference Microscopy as a Technique for Directly Measuring Intracrystalline Transport Diffusion in Zeolites, Microporous Mesoporous Mater. 1999, 32, 101-110.

[11] U. Schemmert, J. Kärger, C. Krause, R. A. Rakoczy, J. Weitkamp, Monitoring the Evolution of Intracrystalline Concentration, Europhys. Lett. 1999, 46, 204-210.

[12] O. Geier, S. Vasenkov, E. Lehmann, J. Kärger, U. Schemmert, R. A. Rakoczy, J. Weitkamp, Interference Microscopy Investigation of the Influence of Regular Intergrowth Effects in MFI-Type Zeolites on Molecular Uptake, J. Phys. Chem. B 2001, 105, 10217-10222.

[13] P. Kortunov, S. Vasenkov, C. Chmelik, J. Kärger, D. M. Ruthven, J. Wloch, Influence of Defects on the External Crystal Surface on Molecular Uptake into MFI-Type Zeolites, Chem. Mater. 2004, 16, 3552-3558.

[14] C. Chmelik, P. Kortunov, S. Vasenkov, J. Kärger, Internal Transport Resistances and Their Influence on Diffusion in Zeolites as Traced by Microscopic Measuring Techniques, Adsorption 2005, 11, 455-460.

[15] E. Lehmann, C. Chmelik, H. Scheidt, S. Vasenkov, B. Staudte, J. Kärger, F. Kremer, G. Zadrozna, J. Kornatowski, Regular Intergrowth in the AFI Type Crystals: Influence on the Intracrystalline Adsorbate Distribution as 
Observed by Interference and FTIR-Microscopy, J. Amer. Chem. Soc. 2002, 124, 8690-8692.

[16] E. Lehmann, S. Vasenkov, J. Kärger, G. Zadrozna, J. Kornatowski, Intracrystalline Monitoring of Molecular Uptake into the One-Dimensional Channels of the AFI-Type Crystals Using Interference Microscopy, J. Chem. Phys. 2003, 118, 6129-6132.

[17] E. Lehmann, S. Vasenkov, J. Kärger, G. Zadrozna, J. Kornatowski, Ö. Weiss, F. Schüth, Inhomogeneous Distribution of Water Adsorbed under Low Pressure in CrAPO-5 and SAPO-5: An Interference Microscopy Study, J. Phys. Chem. B 2003, 107, 4685-4687.

[18] C. Chmelik, E. Lehmann, S. Vasenkov, B. Staudte, J. Kärger, Application of Interference and IR Microscopy for Studies of Intracrystalline Molecular Transport in AFI Type Zeolites, Fluid Transport in Nanoporous Materials, Springer, Dordrecht 2006, 575.

[19] J. Kärger, C. Chmelik, E. Lehmann, S. Vasenkov, Monitoring the Intracrystalline Distribution of Guest Molecules in Zeolites, Stud. Surf. Sci. Catal. 2004, 154, 1791-1796.

[20] J. Weitkamp, L. Puppe, Catalysis and Zeolites, Springer-Verlag, Berlin, Heidelberg 1999.

[21] C. Baerlocher, W. M. Meier, D. H. Olson, Atlas of Zeolite Framework Types, Elsevier, Amsterdam 2001.

[22] J. Kärger, R. Valiullin, S. Vasenkov, Molecular Dynamics under Confinement to One-Dimension: Options of Measurement and Accessible Information, New J. Phys. 2005, 7, 15.

[23] M. Hermann, W. Niessen, H. G. Karge, Sorption Kinetics of n-Hexane in MFI-Type Zeolites Investigated by Micro-FTIR Spectroscopy, in Catalysis by Microporous Materials (Eds: H. K. Beyer, H. G. Karge, I. Kiricsi, J. B. Nagy), Elsevier, Amsterdam 1995, 131-138.

[24] H. G. Karge, W. Niessen, H. Bludau, In-Situ FTIR Measurements of Diffusion in Coking Zeolite Catalysts, Appl. Catal., A 1996, 146, 339-349.

[25] W. Niessen, H. G. Karge, Diffusion of p-Xylene in Single and Binary Systems in Zeolites Inve- stigated by FTIR Spectroscopy, Microporous Mater. 1993, 1, 1-8.

[26] R. A. Rakoczy, Y. Traa, P. Kortunov, S. Vasenkov, J. Kärger, J. Weitkamp, Synthesis of Large Crystal of All-Silica Zeolite Ferrierite, Microporous Mesoporous Mater. 2007, in press.

[27] Y. Long, M. Ma, Y. Sun, H. Jiang, Synthesis, Ion-Exchange, Structural Characterization and Adsorption of K, Na-FER Type Zeolite, J. Incl. Phen. Mac. Chem. 2000, 37, 103.

[28] R. E. Morris, S. J. Weigel, N. J. Henson, L. M. Bull, M. T. Janicke, B. F. Chmelka, A. K. Cheetham, A Synchrotron X-ray Diffraction, Neutron Diffraction, 29Si MAS-NMR, and Computational Study of the Siliceous Form of Zeolite Ferrierite, J. Am. Chem. Soc. 1994, 116, 11849-11855.

[29] H. Gies, R. P. Gunawardane, One-Step Synthesis, Properties and Crystal Structure of Aluminium-Free Ferrierite, Zeolites 1987, 7, 442-445.

[30] P. Kortunov, C. Chmelik, J. Kärger, R. A. Rakoczy, D. M. Ruthven, Y. Traa, S. Vasenkov, J. Weitkamp, Sorption kinetics and intracrystalline diffusion of methanol in ferrierite: An example of disguised kinetics, Adsorption 2005, 11, 235-244.

[31] P. Kortunov, L. Heinke, S. Vasenkov, C. Chmelik, D. B. Shah, J. Kärger, R. A. Rakoczy, Y. Traa, J. Weitkamp, Internal concentration gradients of guest molecules in nanoporous host materials: measurement and microscopic analysis, J. Phys. Chem. B 2006, 110, 23821-23828.

[32] L. Heinke, C. Chmelik, P. Kortunov, D. B. Shah S. Brandani, D. M. Ruthven, J. Kärger, Analysis of Thermal Effects in Infra-Red and Interference Microscopy. n-Butane-5A and Methanol-Ferrierite Systems, Microporous Mesoporous Mater. 2007, in press.

[33] J. Crank, Mathematics of Diffusion, Oxford University Press, Oxford 1956.

[34] J. Kärger, P. Kortunov, S. Vasenkov, L. Heinke, D. B. Shah, R. A. Rakoczy, Y. Traa, J. Weitkamp, Unprecedented Insight into Diffusion by Monitoring the Concentration of Guest Molecules in Nanoporous Host Materials, Angew. Chem. Int. Ed. 2006, 45, 7846-7849. 